\title{
MIGRACIONES INTERNACIONALES: REPENSANDO LAS CIUDADES Y SUS POLÍTICAS.
}

\section{INTERNATIONAL MIGRATION: RETHINKING POLICIES OF THE CITIES.}

https://doi.org/10.17979/relaso.2011.1.1.1197

\section{Carolina Mera (IIGG-FCS-UBA/ CONICET) ${ }^{1}$ Gerardo Halpern (IIGG-FCS-UBA/ CONICET) ${ }^{2}$}

\section{Resumen:}

En el presente artículo proponemos un conjunto de reflexiones críticas acerca del campo de las migraciones internacionales y su relación con las ciudades. Para ello, se postulan una serie de consideraciones acerca de diferentes procesos migratorios contemporáneos -tanto en su dimensión global como local-, la formación de las redes trasnacionales de migrantes, su posibilidad de acceso a derechos o no y las condiciones de igualdad y de desigualdad que atraviesa la relación entre migrantes internacionales y ciudad. Es decir, a partir de una mirada que ancla en la perspectiva del sistema-mundo, se recuperan tres dimensiones fundamentales para la temática: Estados, redes transnacionales y sujetos.

El análisis focaliza en algunas características actuales de las migraciones en la Ciudad Autónoma de Buenos Aires para pensar cómo la dimensión local manifiesta, fuerza o repliega formaciones colectivas -étnicas, nacionales, territoriales- que, además de mostrar la conflictividad estructural de la globalización, resultan objeto de

\footnotetext{
1 Doctora en Antropología Social y Etnología Urbana de la EHESS. Licenciada en Sociología de la UBA. Docente de la UBA. Investigadora de CONICET con sede en el Instituto de Investigaciones Gino Germani de la FCS de la UBA.

2 Doctor en Filosofia y Letras de la Universidad de Buenos Aires. Licenciado en Ciencias de la Comunicación de la UBA. Docente de la UBA. Investigador del CONICET con sede en el Instituto de Investigaciones Gino Germani de la FCS de la UBA.
} 
xenofobia y estigmatización, así como expresión de resistencias y de demandas.

\section{Ciudad - Migración - Sentidos globales y locales}

\section{Abstract:}

In this paper, we suggest a set of critical thinking about international migrations and its relation to the cities. For this, considerations about different contemporary migratory processes -not only globally, but also locally-, the formation of transnational networks, the possibility of access to rights and equal opportunities between international migrants and the city are postulated. That is to say, from a world system perspective, three fundamental dimensions of the subject matter were recovered: States, transnational networks and individuals.

The research centers in a number of current characteristics of migrations in the City of Buenos Aires in order to show how the local dimension displays, forces or withdraws collective formations -ethnics, nationals, territorial. The structural conflict of globalization shown by the collective formations produces not only xenophobia and stigmatization, but also opposition and complaints.

\section{Cities- Migration - Global and local sense.}

\section{Las migraciones y las ciudades}

Nos proponemos reflexionar críticamente sobre lo que podria denominarse el campo de las migraciones internacionales $\mathrm{y}$ su relación con las ciudades. Entendemos que estudiar las nuevas dinámicas de las migraciones a partir de un modelo territorial de relación social debe priorizar el debate acerca de las estrategias de vida, de instalación -en formas de hábitat, salud, inserción 
económica/ laboral y asociativas- como parte de comportamientos socio-culturales que adquieren diferentes modalidades de integración a escala local y global.

Debemos repensar la importancia de las ciudades y de sus políticas en el panorama migratorio contemporáneo: la forma en que el individuo se relaciona con los otros y produce individual y colectivamente las afectividades a partir de las presencias físicas y virtuales, las valoraciones del medio y de las movilidades en los diferentes escenarios (sean estos de partida, temporarios y de llegada), entre otras situaciones, nos llevan a cuestionar algunos presupuestos que a veces se presentan como obvios ${ }^{3}$.

El fenómeno migratorio es de una complejidad que solo puede ser abordada desde la multiplicidad de factores, principalmente aquellos que hacen a la producción de diferentes tipos de migrantes. De manera sumamente esquemática, por un lado están las migraciones que se pueden considerar como "privilegiadas", y que son el producto de la globalización que contiene y promueve movilidades transnacionales: ejecutivos, técnicos de empresas, científicos, artistas, etc., actores sociales que forman parte de un movimiento legitimado y sobre el cual se estructuran buena parte de los relatos que muestran la efectiva concreción de la globalización como sinónimo de armonía e integración. Pero, por el otro lado, están las migraciones que son el producto de crisis económicas, impactos de los programas de ajuste estructural, adversas condiciones sociales debidas al continuo crecimiento de la pobreza y las desigualdades sociales $^{4}$, es decir, aquellas

3 Siguiendo la línea de reflexión de Mato (2007:58), la transnacionalización no necesariamente implica "desterritorialización" en el sentido de falta de referencia al lugar. En general, según el autor, las negaciones de la relevancia de las referencias a los lugares suelen hacerse desde miradas que priorizan el rol de las personas como simples "usuarios" y/ o "consumidores", sin considerar la puesta y producción de sentidos a partir de las relaciones establecidas en/con esos lugares. ${ }^{4}$ Con respecto a la incorporación de estos migrantes a la Ciudad, en lo económico se incorporaron a sectores de baja productividad como la construcción, el servicio doméstico, como trabajadores fabriles con baja calificación (textil, confección y calzado), y en sectores del mercado laboral con condiciones de trabajo muy 
para las cuales la globalización no ha implicado una discursividad de promoción, sino, más bien, una sistemática criminalización, una constante regulación y una escalada de formas de administración que los lleva a ser la expresión del "vagabundo" del que habla Bauman.

Ahora bien, y para evitar cierta seductora mirada dual, si nos atenemos a una fotografia del mapa migratorio regional contemporáneo, veremos que para el 2000, el total de inmigrantes intra-regionales alcanzó, según el Boletín del Observatorio Miurbal (2007), 3 millones de personas, principalmente asentados en ciudades. La mayoría de estos migrantes internacionales se suma a la población urbana de bajos ingresos, encuentra empleo en el sector informal y más vulnerable $\mathrm{y}$, generalmente, tiende a vivir entre la propia comunidad local en asentamientos precarios o informales. Ello nos permite considerar que, si se acepta la analítica esquematización planteada, la gran parte de la migración corresponde al segundo grupo.

Por otro lado, pero en relación con lo anterior, en la Argentina existe muy poca información sobre el acceso a la vivienda, infraestructura urbana, salud, educación, espacios públicos; posibilidad de participación cívica y política de los migrantes ya que, precisamente al tratarse de población vulnerable, pobre, marginalizada la migración es considerada como parte de las poblaciones pobres, beneficiarias de planes universalistas, homogeneizadas en totalidades que operan, como dice Caggiano (2005), como discriminaciones por indiscriminación.

En contraposición, aquellas migraciones "privilegiadas" están regidas por acuerdos privados transnacionales de ciertos aspectos de la regulación de la movilidad laboral (Sassen, 1998) y se desarrollan en otros circuitos poblacionales, laborales, urbanos. Incluso, si bien tampoco

precarias. La misma vulnerabilidad se encuentra en las situaciones que viven los migrantes en ámbitos residenciales y en el área de salud. 
se posee una dosis de información muy significativa, no deja de ser cierto que su acceso a derechos se encuentra mucho menos limitada que en el grupo anterior, pues se trata de un sector que no solo posee diversos tipos de capitales para acceder a los mismos, sino que, además, inscriben sus procesos migratorios dentro de tramas institucionales menos burocratizadas, menos limitadas y limitantes. En cierta manera, se trata de migrantes que, en general, no atraviesan los mismos problemas que el otro grupo.

Dado que consideramos que la migración internacional es cada vez más un fenómeno urbano debemos aceptar también la necesidad de su inclusión en la agenda de las ciudades y de los gobiernos locales. Sin embargo, a pesar de lo dicho la migración sigue siendo considerada como parte de una cuestión de seguridad nacional, de control de la circulación de las personas (egreso e ingreso) y, en el mejor de los casos, desconsiderada para su gestión. En muy raros casos existe una política para migrantes internacionales que prevean su inclusión urbana y fomente dinámicas interculturales $\mathrm{y}$ de igualdad. $\mathrm{Y}$ no nos referimos sólo a políticas sociales sobre educación, vivienda, salud, sino también a planes de alfabetización para adultos, promoción de la cultura, revalidación de títulos, acceso a la información.

Aun así, las migraciones, sean privilegiadas o vulnerables, se articulan en redes familiares, religiosas, asociativas, étnicas, empresariales que se ponen en tensión en el escenario de la ciudad a la vez que plantean diferentes demandas al Estado (sobre todo de destino, aunque habria que considerar también al de origen). Estas demandas, a su vez, están condicionadas por el nivel de organización de cada grupo migrante. Pertenecer a una diáspora (como los chinos, coreanos, hindúes, griegos) o simplemente a redes migratorias consolidadas en el tiempo (como los bolivianos, paraguayos, peruanos, ecuatorianos e, incluso, 
senegaleses) brinda un conjunto de ventajas en relación a otras migraciones más recientes y/o más dispersas.

En todo caso, cuanto mayor despliegue de redes y mayor organización comunitaria, menos demandan al Estado local y Nacional en relación con sus necesidades inmediatas (vivienda, trabajo), puesto que logran mitigarlas circunstancialmente por fuera de los esquemas institucionales que, como decíamos arriba, realizan un escaso aporte para su resolución.

Dichas redes, en todo caso, posibilitan ampliar los márgenes de demanda sobre áreas más complejas, como ser los derechos políticos, la modificación de normativas, etc. Es decir, así como esas redes logran "contener" reclamos, a la vez se constituyen en actores de reclamos ante los Estados. Ello conlleva la particularidad de que, así como logran promover espacios colectivos de organización, esos espacios devienen ámbitos de mejora de la situación de los migrantes. En ese sentido, muchas de estas formaciones colectivas terminan funcionando alli donde el Estado no reconoce o no promueve la concreción de derechos. Estas redes, entonces, cumplen funciones que resultan beneficiosas para los migrantes así como para los Estados.

Ello no supone que la demanda de acceso a derechos desaparezca, sino que, más bien, la reconduce a través de las organizaciones que se constituyen $o$ se pueden constituir en actores políticos que, según el caso, asumen posiciones públicas de demanda que involucran a los Estados. Esas redes institucionalizadas se convierten en grupos de presión que interpelan a los Estados de origen y de destino de diversas maneras.

Estos procesos colectivos explican en gran parte muchos de los que son denominados "éxitos migratorios", incluso a pesar de las políticas de fronteras cerradas, las implicancias de vivir como indocumentados, las dificultades en el acceso a la salud y al empleo. Las redes 
operan, en uno de sus niveles, como contrapeso de situaciones adversas y posibilitan su reproducción, crecimiento y, a veces, su mayor o menor formalización.

¿Qué aportan las redes a los migrantes y a sus comunidades, y de qué manera contribuyen a la vida de las ciudades de partida y de destino? En el presente trabajo no intentamos dar respuestas sino simplemente aportar a esta reflexión desde una mirada crítica sobre algunos aspectos de estos procesos migratorios que se invisibilizaron, permitiendo la idealización de otros aspectos parciales del mismo proceso que se idealizaron.

\section{Dimensiones de análisis}

Para analizar el desplazamiento de personas -con diferentes competencias de calificación- y su impacto sobre las ciudades involucradas es importante dar cuenta de diferentes dimensiones.

Priorizamos una mirada estructural del sistema mundo (Wallerstein, 2001) que pone de relieve los enfoques macroeconómicos $\mathrm{y}$ donde se hacen relevantes los desequilibrios regionales. Esta perspectiva se complementa con la perspectiva transnacional (Sassen, 2003; Ortiz, 2003) a partir de la cual adquiere nueva significación e importancia el concepto de redes. Esta perspectiva abre la observación de los procesos migratorios a las dinámicas de los mismos en sus dimensiones locales. Y ello nos permite dar cuenta de la conformación de subjetividades, la producción de motivaciones, y la elaboración de expectativas.

Ahora bien, en la situación actual del sistema mundo es imprescindible dar cuenta de estos aspectos para poder recuperar la capacidad reflexiva sobre los desplazamientos y su aporte a los procesos de desarrollo de los países de origen y de residencia. 
Tanto las estructuras políticas, económicas y culturales de los estados nacionales involucrados, como la transnacionalización de las sociedades y las dinámicas de los sujetos individuales son parte de una articulación que incide en los individuos y actores colectivos de los respectivos anclajes locales y juegos globales.

1) Son las estructuras y gestiones de los estados nacionales y locales las que promueven e invierten en educación y cultura, salud, vivienda, seguridad y condiciones de vida de los ciudadanos de los países en desarrollo. En este sentido, según cómo se perciba el fenómeno migratorio, se tomarán o no medidas correspondientes. Por ejemplo, mientras que en América Latina los migrantes entran dentro de las poblaciones carenciadas contempladas con medidas de inclusión socioeconómicas -en general universalistas-, en Europa son muy tenidos en cuenta en lo que politicas culturales atañe, ya que se percibe como un problema de integración sociocultural $^{5}$.

2) La transnacionalización de las vidas a partir del abaratamiento de las comunicaciones y el transporte, las nuevas formas de comunicación que ofrece Internet, la internacionalización de la economía, del sistema de educación superior, del arte, también deben ser considerados críticamente como nuevas dinámicas de la desigualdad. Se habla de la transnacionalización del capital, de las empresas pero, para hablar de su contraparte, la migración, es muy raro que se lo haga como transnacionalización de la mano de obra. (Sassen 1998). Esto no significa que compartamos la idea de que la migración y los medios de comunicación marcan el desborde de la modernidad (Appadurai, 2001), sino más bien que son fenómenos cada vez más inescindibles y que se atraviesan mutuamente. Pero que, a la vez, no han implicado el fin de los Estados nacionales sino más bien lo

${ }^{5}$ De hecho dan cuenta de estas preocupaciones el protagonismo de los migrantes y sus descendientes en las movilizaciones urbanas de los últimos años. 
contrario. Así como los Estados han reaccionado con políticas fuertemente anti-inmigratorias, los migrantes -no solo a través de sus redes, pero también a través de ellashan desarrollado fuertes politicas identitarias que reponen al Estado nacional en el dentro de sus autopercepciones (Levitt y Glick Schiller, 2004).

3) Las dinámicas de movilidad que adoptan los sujetos también se ven condicionadas por situaciones que se intensifican cada vez más como el acceso a otros idiomas, el incremento del turismo y conocimiento de otras formas de vida, la existencia de redes ya establecidas entre ciudades y países, las nuevas formas de sociabilidad de la Web, la creciente internacionalización de los mercados laborales para las personas altamente calificadas. Estas prácticas, cada vez más valoradas, generan movilidades y estilos de vida diferenciados, donde el valor positivo está cada vez más puesto en algunos tipos de movilidad asociados a las nuevas formas globales del poder. Si la nueva extraterritorialidad de la elite huele a libertad embriagadora, la territorialidad del resto huele cada vez menos a hogar y más a prisión (...) (Bauman 1999:35).

Además, desde el imaginario social (y muchas veces en estrecha relación con las políticas que señalamos en el primer punto) se sigue condenando a las comunidades migrantes que establecen sus propios barrios y son acusados de "no integrarse a la sociedad", acusación que evidencia una pretendida pretensión asimiladora; se sigue condenando a las comunidades migrantes por "mantener sus costumbres", muchas veces estigmatizadas y estereotipadas, se sigue condenando a las comunidades migrantes de promover sus relaciones intraétnicas, desacreditadas y exotizadas; condenas que delimitan no solo la relación entre "nosotros y los otros" sino que, en su mismo acto define la significación del otro, su abyección y su irremediable alteridad constitutiva. Sin embargo, no se habla acerca de que es precisamente gracias a esas redes, a esas territorialidades, costumbres, relaciones 
intraétnicas, que estos grupos pueden sobrevivir en un sistema internacional y local de producción de desigualdad y pobreza.

\section{Relocalizar el debate. Los migrantes presentes y ausentes de la "ciudad"}

Creemos necesario aquí discutir varios mitos presentes en ciertos discursos hegemónicos que tienen que ver con los beneficios que las redes aportan al fenómeno y las valoraciones de la movilidad. Ambas entradas, a su vez, se han constituido en eje de las definiciones de políticas y propuestas de los organismos internacionales que han tomado a la "cuestión migratoria" como parte de sus agendas planificadoras.

En cuanto a la movilidad, existe un doble discurso: por un lado se valora cierta movilidad actual que tiene que ver con el estilo de vida de la "globalización" y se denigran otras, relacionadas justamente con las desigualdades y exclusiones regionales. El valor de la movilidad, en los procesos locales de instalación de las migraciones forma parte de la dinámica global-local, de lo cual depende la posibilidad de desplazarse con mayor o menor éxito a través de la red global.

En el nuevo mundo de la posguerra por el espacio, la movilidad se ha convertido en el factor estratificador más poderoso y codiciado de todos; aquél a partir del cual se construyen y reconstruyen diariamente las nuevas jerarquías sociales, políticas, económicas y culturales de alcance mundial. Y a los que ocupan la cima de la nueva jerarquía, la libertad de movimiento les otorga muchas más ventajas (...) (Bauman, 1999: 16-17). Asi, "quien tenga libertad para escapar de la localidad, la tiene para huir de las consecuencias. Este es el botín más importante de la victoriosa guerra por el espacio" (Bauman, 1999: 16). Morley parece acordar con esta postura al sostener: "cada vez con mayor frecuencia la inmovilidad es vista como una 
de las formas de empobrecimiento" (2005: 139). Cabe entonces preguntarse quién tiene el control sobre las movilidades. Gran parte de los trabajadores en desventaja en las ciudades globales son las mujeres, los inmigrantes y las personas étnicamente diferentes, cuyo sentido político de pertenencia y cuyas identidades no están necesariamente incorporadas a la nación o a la comunidad nacional (Sassen, 1998). Son parte de un mismo proceso de globalización: financiera y empresaria por un lado, y de exclusión y marginación por otro.

Mucho se ha escrito acerca del impacto de las migraciones transnacionales ${ }^{6}$ en las ciudades involucradas y en la vida de los migrantes y sus familias. En el caso de los migrantes, si bien son considerados como "problemas" (de hecho se habla del "problema migratorio"), se percibe el beneficio de las redes en el proceso de desplazamiento y en su calidad de vida. En definitiva, su contribución para que los migrantes se desenvuelvan sin la ayuda del Estado, como por ejemplo en lo que refiere a "los indocumentados", pareciera ser el final feliz de un relato que ocluye la desigualdad estructural que los atraviesa. Ya no habria tanta significación en el acceso diferenciado a derechos, dado que, en realidad, el problema deja de ser tal gracias a la conformación de las "comunidades". Ello, en definitiva, dispensa a los Estados de asumir sus obligaciones respecto de la igualdad que, por ejemplo, la Constitución argentina reserva para todos los habitantes del mundo que quieran habitar el suelo argentino.

Se suma a ello que esos Estados naturalizan su producción de alteridad y desigualdad, ubicando a los migrantes como disrupciones de una armonia que se ve trastocada por la presencia del "extranjero". En ese

\footnotetext{
${ }^{6}$ Sobre todo si concebimos las redes como configuraciones de un campo - en el sentido que le da Bourdieu- constituido por posiciones de los actores, intereses y conflictos, relaciones de poder, que legitiman tanto las temáticas y titulaciones, como la dirección de los financiamientos y de las movilidades, etc. con mayor o menor nivel de tensiones y negociaciones.
} 
sentido, las ciudades, en primer término, son percibidas también desde el "problema" que acarrean estos migrantes, especialmente por la sobrecarga de nuevas demandas sociales de habitación, salud, educación, que estos parecieran acarrear.

Sin embargo, estos procesos son considerados desde parcialidades que obliteran un análisis más complejo, donde se destacan variables ausentes como los beneficios que el migrante trae a la pretendidamente homogénea ciudad en la que se establecen, sus aportes culturales, económicos y políticos; $\mathrm{y}$, de otra parte, no son considerados los perjuicios que ocasionan en la ciudad que dejan, tanto a nivel social-comunitario como individual y psicológico. En general los enfoques de la relación ciudadmigrante se concentran en la ciudad de residencia (receptora) y no en la ciudad familiar, de historia y de afectos, "home".

Notemos con más detalle algunos casos donde también se expresan solo algunos factores parciales del fenómeno y que dejan de lado precisamente aquellos que hacen a los efectos de la "localización", como el de las redes de científicos, o el de los beneficios de las remesas:

Algunos estudios recientes sostienen que el científico o el profesional emigrado puede ser una contribución al desarrollo del país de origen gracias a la potencialidad de las redes de intercambio. Esta concepción deja de lado la dimensión fundamental de lo local. En la práctica local intervienen distintas dimensiones: por un lado, interactúa e influye en cadenas de relaciones sociales con colegas, estudiantes, proveedores, familiares, amigos, vecinos, etc. Por otro lado, se desempeña en una estructura material de universidades, centros, laboratorios, instrumentos, documentos, bibliografias, etc. Esta presencia local crea mejores condiciones para una apropiación del saber por parte de la sociedad y sobre todo para que la eficiencia innovativa se potencie con sectores del sistema colectivo de 
creación y uso de conocimientos. Las políticas de investigación e innovación y desarrollo deben tener muy en cuenta el "efecto de localización", es decir las características específicas de las economías locales y las relaciones socio culturales e institucionales de las mismas ${ }^{7}$.

Lo mismo podríamos decir con respecto al aporte de las remesas. Si bien no queremos discutir el impacto de su beneficio en ciertas economias, lo que nos interesa es reflexionar sobre los aspectos perjudiciales para la ciudad y los grupos que allí residen.

La inclusión de la dimensión local permite dar cuenta de complejidades que afectan la calidad de vida de las familias, grupos y ciudades. Al igual que el científico mencionado anteriormente, el ciudadano en presencia aporta cotidianamente algo irremplazable a la cultura económica, social y política del lugar. Genera relaciones afectivas y de transmisión de saberes que hacen a las formas narrativas mediante las cuales las sociedades construyen sus historias e identidades. Su ausencia genera familias y grupos fragmentados, distanciados, que deben afrontar nuevos problemas psicológicos y de sociabilidad.

\footnotetext{
${ }^{7}$ Siguiendo esta línea de reflexión, consideramos que sigue actual la concepción de la emigración de recursos humanos calificados - tan estudiada durante las década del 50 al 70 bajo el título de "fuga de cerebros" (Oteiza; 1976)-, que prioriza la pérdida ocasionada en los países con menor desarrollo en beneficio de los países industrializados. Estos análisis dieron prioridad al tiempo y recursos materiales que el país con menor desarrollo había invertido en la formación de aquel profesional o estudiante, perdiendo además de recursos humanos, su creatividad, su energía y su talento, además de la potencialidad que estos recursos significan en el proceso de producción y acumulación de conocimiento (lo cual sigue siendo muy dificil de medir). Como confluencia del desarrollo tecnológico y de la reestructuración del mercado de trabajo producto de las políticas neoliberales representadas en el consenso de Washington-, se crean vacancias en la estructura ocupacional en las áreas de ciencia, tecnología y comunicación de los sistemas productivos de las economías del Norte. De esta manera la fuga de cerebros o brain drain aparece en la agenda política internacional, pero esta vez etiquetada como circulación de cerebros. Sin embargo, y a riesgo de ser acusados de sesentistas tenemos que poder pensar la emigración desde los impactos desiguales en los procesos de desarrollo, y que si bien podemos hablar de circulación de talentos entre sistemas científicos y tecnológicos consolidados, no podemos hacerlo cuando el flujo se produce entre sistemas desiguales.
} 
También a nivel comunidad podemos ver impactos, como las nuevas formas de jerarquías de poder construidas a partir de los ingresos de los emigrados.

La mayoria de los estudios migratorios se concentran en la vulnerabilidad en la que se encuentra la población migrante urbana en las ciudades de residencia y dejan de lado las consecuencias humanas que estas movilidades producen en los contextos de salida. Esta tendencia se debe, en primer lugar a que se prioriza el análisis causal de la situación socio-económica vinculada a las consecuencias de los modelos de desarrollo poco integradores y tremendamente expulsivos de mano de obra, la concentración del ingreso, a la desocupación, la informalidad y la precariedad en el empleo, y su paralelo: la capacidad de ciertas economias de incorporarlos (de forma más o menos precaria y vulnerable). En segundo lugar, podríamos mencionar la precariedad en el ejercicio de derechos de tipo político como protección policial, acceso a la salud, la educación, o al trabajo que estos migrantes sufren en los dos contextos.

\section{Los migrantes en la Ciudad de Buenos Aires}

Según los recientes datos del Censo de 2010, la población total de Argentina es de 40.117.096 habitantes. La población extranjera alcanza 1.805.957, un 4,5\% de la población total, es decir, apenas $0,3 \%$ más que en 2001 cuando se produjo el menor porcentual de la historia del país. El 3,5\% de la población proviene de países limitrofes y del Perú (1.402.568 habitantes) mientras que el 0,7\% de Europa (299.394 habitantes).

$\mathrm{Si}$, por un lado, la Ciudad y la Provincia de Buenos Aires (CABA y PBA, respectivamente) albergan al 46\% de la población total del país, dicha región, para el caso de los migrantes internacionales alberga al 73,3\%. Es decir, que el comportamiento de asentamiento de los migrantes repite y profundiza el del modelo de concentración poblacional 
que impera en el país y que manifiesta cada vez más patrones urbanos.

Si recortamos solo a la migración proveniente de los países limitrofes y del Perú, dicha concentración es del 71,68\%. El desglose por lugar de nacimiento también muestra algunas particularidades, como ser que casi el $86 \%$ de los paraguayos, el $66 \%$ de los bolivianos y el $82,5 \%$ de los peruanos se concentran en la CABA y la PBA. Este proceso de metropolización, que ya habia sido registrado por el INDEC hacía veinte años, pareciera profundizarse en algunos casos hasta niveles absolutos (los casos de paraguayos y peruanos son emblemáticos de ello).

El actual amesetamiento de población extranjera en la Argentina -y que se desprende de los datos relevados- se debe, básicamente a dos variables: la primera es la permanencia de un flujo migratorio regional constante que, a lo largo de la historia argentina se ha mantenido en índices más o menos cercanos al 3\%. El actual 3,5\%, si bien permite apreciar un incremento porcentual de inmigrantes regionales respecto del componente poblacional nativo (y, lógicamente, también respecto del total de extranjeros), también muestra cómo -aun así- se mantiene constante su proporcionalidad y, junto con ella, su histórica escasa significación.

Sí resulta más relevante el segundo aspecto: la disminución porcentual de europeos en el país, lo que se debe, centralmente, a la defunción de la longeva composición de esa migración y la no renovación de ese stock migratorio. Por ende, si la migración regional se mantiene más o menos constante, la migración europea es cada vez más pequeña.

De ahí que los guarismos adquieran proporciones interesantes, sobre todo en contraste con la historia del país y con su épica migratoria, sintetizada en que "los argentinos descienden de los barcos": casi 78 de cada 100 extranjeros en la Argentina de 2010 provienen de países de 
frontera y del Perú, mientras que no llegan a 17 de cada 100 los provenientes de Europa.

Dada la especificidad de este trabajo, brindaremos algunos datos de bolivianos, paraguayos y peruanos, pues, además de ser -junto con los chilenos- los grupos migratorios más significativos, son los que han sido más visibilizados en los últimos años en el país a partir de una discursividad xenófoba que los ha puesto en la agenda política, pública y mediática como "masivos e invasivos" (incluso, a pesar de lo que la evidencia estadística y académica muestran) (Halpern, 2009). Por otro lado, a diferencia de los chilenos (que retroceden un $10 \%$ ), se trata de los grupos más numerosos que describen importantes incrementos intercensales entre 2001 y 2010 (48\% los bolivianos, 70\% los paraguayos y $80 \%$ los peruanos) y que, junto con ello, concentran la discursividad contemporánea respecto de las migraciones en el país. Con esto no estamos diciendo que la cantidad determine la discursividad -seria absurdo empírica y analíticamente sostener eso- sino que es la discursividad la que condensa determinados imaginarios y que construyen diferentes cuantificaciones. En ese sentido, esas "cuantificaciones" son operaciones discursivas $\mathrm{y}$, como tales, operaciones ideológicas de legitimación de determinadas formas de la desigualdad social. Marcamos esto porque en materia migratoria si bien es importante trabajar con los datos que se poseen, no es menos cierto que se trata de un campo en el que la producción de números se ha vuelto un fetiche desde el que, en general, se legitiman las prácticas y políticas xenófobas. La referencia a este momento histórico como el de "las migraciones internacionales" (tanto por parte de importantes autores como del discurso de los organismos internacionales y de los aduladores de la denominada "globalización") pareciera recostarse sobre el supuesto de que vivimos una explosión migratoria mundial. Sin embargo, no hay una sola evidencia que permita sostener esa afirmación. De todos modos, se ha vuelto una verdad 
indiscutible. Es decir, tenemos una verdad indiscutible que es... falsa. Pero esa "verdad" se estructura en torno al número que imagina y proclama, con una efectividad similar a la que operaciones discursivas iguales se efectúan en la Argentina respecto de las migraciones regionales. En este plano "cuantificación" y "dato" no significan lo mismo.

La referencialidad contemporánea a bolivianos, paraguayos y peruanos se acerca más a las formas de visibilización en contextos de desigualdad de ciertas migraciones y de ciertos componentes fenotípicos que se les asocian que a novedosos procesos inmigratorios en la Argentina. Esto último, además, supone una clara relación con las condiciones materiales (que implican, socioculturales) de producción de esas específicas discursividades, las cuales se producen, precisamente, en Buenos Aires, ese sesgado panóptico del que habló Pacecca (2000) y que, como elaborador discursivo emblemático de la Argentina, construye una extranjerización de determinados componentes sociales.

Dos aclaraciones al respecto: la primera es que dicha extranjerización -motivada por factores que no analizamos en este trabajo- es la que posibilita la distorsión perceptiva inherente a los marcos xenófobos. Es decir, se ven muchos más extranjeros que los que hay (sin adentrarnos aquí en los problemas que ello supone e interpela); la segunda aclaración es que el hecho de que focalicemos en Buenos Aires como productor xenófobo "nacional", no significa que no existan diversas formas, llamémosle, locales de xenofobia. Es decir, la extranjerización que se produce en Buenos Aires puede coincidir o no con otras tantas formas de producción de alteridades desfavorables en la Argentina. Así, determinadas cargas fenotípicas en Buenos Aires se constituyen en marcaciones de alteridad, mientras que en otras zonas del país son otros elementos los que se ponen en juego o los que producen esas alteridades. Con ello queremos decir que, así como somos críticos de las formas 
xenófobas de Buenos Aires, no creemos que las demás variantes de construcción de alteridades desfavorables sean mejores, ni mucho menos graves.

Ahora bien, y volviendo sobre algunos datos que permiten hacer ciertas conjeturas, si bien es necesario profundizar en el análisis de los datos del censo 2010 de la Argentina, debemos aclarar que al momento de escritura de este texto solamente se cuenta con los datos generales de población, no así con unidades más detalladas que nos permitan reponer más elementos. Por ende, las caracterizaciones que aportamos a continuación refieren a datos anteriores e investigaciones de colegas que se han adentrado en la elaboración de bases propias o bien en el análisis de la Encuesta Complementaria de Migración Internacional (ECMI) que se hizo entre 2002 y 2003 como consecuencia del censo 2001.

También debemos aclarar que, si bien no haremos detallados abordajes de cada una de estas corrientes migratorias, el proceso de cada uno de estos colectivos no es homogéneo. $\mathrm{Ni}$ en términos históricos ni en términos sociolaborales o de áreas de inserción laboral.

Así como se pueden destacar similaridades, no es menos cierto que, según la especificación del abordaje, se pueden encontrar significativas diferencias, por ejemplo, entre varones $\mathrm{y}$ mujeres; entre migrantes bolivianos $\mathrm{y}$ paraguayos; entre corrientes provenientes de un mismo país en diferentes momentos históricos, etc.

En lo que refiere a las áreas económico-laborales, estos migrantes se incorporan a sectores de baja productividad como la construcción, el servicio doméstico, trabajadores fabriles con baja calificación (textil, confección y calzado), en sectores del mercado laboral con condiciones de trabajo más peligrosas y vulnerables (explotación, no respeto de legislación laboral).

En un reciente trabajo de Alicia Maguid y Sebastián Bruno sobre migración boliviana y paraguaya en el AMBA (Área 
Metropolitana de Buenos Aires), los autores afirman que "Las ramas de actividad que absorben a estos migrantes difieren de acuerdo con origen y sexo, pero una vez más se comprueba la persistencia de un espectro limitado de sectores, donde se encuentran verdaderos nichos que concentran a la mano de obra de estos dos países. El caso más paradigmático de esta situación es el de las mujeres paraguayas, cuyo destino ineludible parece ser el servicio doméstico" (2010: 16). Los autores, tras analizar los datos censales, concluyen que "Más del $80 \%$ de los bolivianos y el $75 \%$ de los paraguayos trabajan en condiciones precarias. Varones y mujeres de ambos orígenes denotan altísimos niveles de precariedad, que alcanzan valores extremos entre las mujeres: $95 \%$ de las bolivianas y $86 \%$ de las paraguayas" (2010: 18)

En una misma línea, Cerruti y Maguid (2005) muestran que 4 de cada 10 trabajadoras de países limitrofes y peruanas estaban empleadas en el servicio doméstico, y que, al $80 \%$ de estas trabajadoras no se le realizan aportes jubilatorios.

En el mismo estudio se señala la subrepresentación de los migrantes en actividades calificadas $(2,4$ contra 9,7 de resto de los trabajadores) y la sobrerepresentación en ocupaciones no calificadas (58,2 contra 31,4 del resto).

En base a la Encuesta Permanente de Hogares de 2005, ambas autoras muestran que, en lo que refiere a la remuneración, mientras en las actividades no calificadas los migrantes limitrofes y nativos obtienen casi el mismo salario, en las actividades calificadas con educación media, la brecha salarial se agranda en detrimento de los migrantes.

Por otro lado, estas autoras, en coincidencia con el resto de los investigadores que han analizado la situación de los trabajadores inmigrantes regionales en el pais, han mostrado cómo la precariedad se agudiza por las condiciones legales, la falta de contrato o la carencia de 
documentos en regla de los inmigrantes. Esta situación los expone a discrecionalidades laborales, además de su evidente impacto en las áreas de salud, vivienda y educación. El hecho de que un inmigrante esté en situación irregular de documentación limita considerablemente su situación y sus alternativas de ejercicio de derechos, dado que queda expuesto a trabajar de manera irregular, no poder realizar aportes previsionales y carecer de obra social.

Ello, además de implicar una tasa de ganancia mayor por parte del capital a expensas del trabajador, significa un conjunto de riesgos objetivos y subjetivos que vive el migrante y ante los cual carece de representación sindical que lo proteja.

Posiblemente como parte de esa situación, la relevancia de las redes comunitarias asume un tipo de especificidad en lo que hace a la contención de los nuevos migrantes. De hecho, muchas de las comunidades logran su inserción (más o menos precaria) movilizando el capital social de las redes de los migrantes, lo cual define la existencia de un capital que -al menos en algunos contextos- opera como condición de posibilidad de producción y reproducción para el migrante. Así, algunas redes pueden establecer nichos de concentración -sea en lo textil, en la alimentación, en la producción y comercialización de hortalizas, en la venta ambulante, en el servicio doméstico, en el cuidado de personas mayores o enfermería, en la construcción, etc.- dependiendo esto de la intensidad de las relaciones y solidaridades étnicas o nacionales.

En ese caso, si bien las redes operan como contenedoras y garantes de la reducción de vulnerabilidades, también se constituyen en estructurantes de los limites de expectativa para los migrantes, al menos en lo que hace a la diversificación de áreas de inserción. La movilidad queda reducida al marco que las redes poseen, lo cual redunda 
en la limitación de las libertades de "circulación" sociolaboral de los migrantes.

Evidentemente, la problemática acá marcada es mucho más compleja que lo que puede mostrar un ensayo, pero sirve para problematizar ciertos límites que poseen las "redes" y que, en su glorificación acrítica, se pierde de vista que un migrante, si bien las necesita, recrea, fortalece y participa, también se ve constreñido a un conjunto acotado de posibles expectativas o alternativas. $Y$ que, como decíamos más arriba, desentiende al Estado respecto de la igualdad de derechos y oportunidades que debería garantizar al total de la población.

En cuanto a la inserción en el espacio urbano, en general los migrantes se instalan en zonas periféricas o en barrios de poco valor económico. Constatamos que ciertos grupos (como los bolivianos) tienden a concentrarse en barrios construidos por ellos mismos a partir de la ayuda comunitaria y en general tienen nominaciones particulares (por ejemplo, Barrio Charrúa). Los residentes del Perú o los más recientes provenientes de África (Senegal), en cambio, se instalan dispersamente en barrios más céntricos de la ciudad como ser San Telmo o San Cristóbal, mediante la ocupación de edificios no habitados o en hoteles.

Los residentes de Chile y Paraguay no tienen barrios propios, tal vez por las caracteristicas de esas migraciones, pero en cambio sí cuentan con asociaciones ligadas a los migrantes por causas politicas y otras. En el caso paraguayo, de todos modos, se evidencian algunas desigualdades urbanas respecto de su distribución en la CABA. Ciertas villas de emergencia constituyen lugares de sobrerrepresentación de paraguayos en relación con el resto de la población y en relación con los paraguayos. Es decir, tanto la villa de Retiro (Villa 31) como la de Barracas (Villa 21) constituyen dos de los emplazamientos más marcados de paraguayos en la CABA. 
Los migrantes coreanos adoptan una fuerte tendencia a reagruparse en barrios, también nominados como Baek$\mathrm{ku}$, a través de la modalidad de repoblamiento en área periférica. Los chinos construyen redes étnicas en lo económico; sin embargo, si bien existe un "Barrio Chino" (en Belgrano C), se trata de un emprendimiento comercial de ciertos grupos chinos y taiwaneses y del gobierno de la Ciudad, dirigido hacia poblaciones no chinas. Los residentes de esta nacionalidad en general no viven en ese barrio sino que hacen uso de este cotidianamente.

\section{A modo de algunas reflexiones...}

Ante la ausencia de políticas que los contemplen como beneficiarios y como sujetos de derechos, los migrantes y sus comunidades tienden a organizarse de acuerdo al potencial de sus redes. Algunas, pertenecientes a procesos diaspóricos, con anclajes locales en las ciudades globales Buenos Aires, San Paulo, México D. F., París, Nueva York, Chicago, Los Ángeles, Seúl y Tokio- permite el acceso a la libertad de movilidad ( $\sin$ ser ejecutivos, estudiantes de elite o de artistas y turistas). Pero sobre todo permite llevar adelante el proyecto migratorio y de vida sin recurrir directamente al Estado, lo que no quita que este sea objeto de demandas por parte de diferentes asociaciones de migrantes. Testimonio de esto es la vida de millones de migrantes indocumentados en Europa, USA o en ciertos países de América Latina, como Argentina.

La propia estructura de la red los incorpora a la ciudad receptora sin asimilarlos (a la vieja usanza), conservando una fuerte pertenencia identitaria, que incomoda como dice Appadurai ciertas seguridades de la modernidad. En este sentido, cada vez más las redes adoptan modalidades de instalación caracterizadas por la concentración en barrios propios o bien de referencia para el encuentro y la institucionalización de espacios que son vividos como propios. A su vez, los comportamientos en el área 
económico-laboral y familiar permiten explicar la reproducción étnica como la dimensión fundamental de la lógica de concentración.

Este tipo de inserción genera (y es generado por) imaginarios (y prácticas) negativos en otros grupos de la Ciudad, a la vez que desresponsabiliza a los gobiernos locales y nacionales de la vida de estas personas en tanto migrantes.

¿Qué relación existe entre estos procesos de redes de migrantes cada vez más organizadas y los miedos que genera la migración?

Las organizaciones en redes transnacionales en el contexto actual nos permiten comprender en la lógica del migrante el por qué de:

1) la segregación socio-residencial, y

2) la adhesión étnica (indirectamente cuestionadora de la identidad nacional o su homogeneidad).

Por otra parte, el proceso global de producción de desigualdad y exclusión alumbra, en la lógica de los discursos hegemónicos el por qué de:

1) los comportamientos y discursos sobre la violencia y el crimen que discriminan y criminalizan a la migración, y

2) los discursos que culpabilizan al migrante por la falta de empleo, la invasión del espacio público, de servicios públicos como salud, educación, asistencia social.

Esto es posible en la medida que el migrante aparece deshumanizado, ajenizado, usufructuario de redes y remesas, y no como parte de procesos colectivos de producción de sentidos y prácticas locales-globales.

Si tenemos en cuenta al migrante en este último sentido, es pertinente preguntarse qué políticas puede pensar la Ciudad para los migrantes? ¿Debe considerárselos parte de una población vulnerable especial o deben ser incluidos y garantizados sus derechos a través de las politicas 
universalistas hacia los niños, ancianos, mujeres, jóvenes, desempleados, etc.? ¿Es posible postular o definir politicas que contemplen la universalidad y la especificidad que expresa el individuo o un colectivo? Incluso, cabe la pregunta acerca de cuál es el alcance de una concepción que sostiene la migración como "derecho humano".

Desde la perspectiva del sistema mundo (Wallerstein, 2001), las migraciones internacionales interpelan y se constituyen en función de estas tres dimensiones (Estados, redes transnacionales y sujetos) implicando cierta naturaleza transgresora de las mismas a partir del cuestionamiento a las fronteras territoriales, culturales e identitarias. Esta trasgresión tiene como escenario privilegiado a las ciudades. En este sentido se abren algunos ejes de reflexión que consideramos imprescindibles para recuperar el debate desde una visión crítica.

La ciudad, en este plano, permite la emergencia de actores, de sus demandas, de su inherente conflictividad. Los gobiernos locales y sus politicas son parte de este juego y de su sensibilidad al tema (y de las tensiones que lo atraviesan) depende que se lleven a cabo políticas para los migrantes o simplemente se siga el juego de las politicas de la nación y el migrante sea considerado un problema que no le corresponde.

Esto supone, además, un desafio respecto de los actores involucrados en el tema. El hecho de que el llamado "problema migratorio" se circunscriba a las redes y la administración de los extranjeros recorta el análisis a un terreno que, cada vez más es puesto en discusión por las dinámicas y actores políticos que asumen la "problemática migratoria" como estructural. Es decir, la reivindicación del acceso a derechos encarnada por organizaciones de migrantes ha mostrado un rasgo novedoso que debe ser tenido en cuenta: la solidaridad de amplios grupos que rechazan las xenofobias locales. En este sentido, el campo 
social en el que la xenofobia aparece como relato hegemónico también encuentra resistencias por parte de algunos sectores de la sociedad local que ponen en cuestión la división "nacional" / "extranjero" como explicación de la temática. Eso permite comprender que ciertos emergentes de politización y reivindicación de derechos que involucran a extranjeros no sean expresados solamente por sectores o agrupaciones de inmigrantes sino también por espacios que promueven una discursividad alternativa a las clásicas concepciones que ubican a la migración dentro de las politicas de seguridad nacional.

En ese marco, el migrante es invocado como parte de las relaciones sociales y de la conflictividad que trasciende la cuestión nacional y lo ubica dentro de la legitimidad del acceso a derechos como parte de la totalidad social.

\section{Bibliografia}

Amsden, A. H. (1989). Asia's next giant. South Korea and Late Industrialization. New York: Oxford University Press.

Appadurai, A. (2001): La modernidad desbordada. México: Trilce - Fondo de Cultura Económica.

Arfuch, L. (2005): Pensar este tiempo. Espacios, afectos, pertenencias. Buenos Aires: Paidós.

Bauman, Z. (1999). La globalización. Consecuencias humanas. Buenos Aires: Fondo de Cultura Económica.

Bauman, Z. (2005). Identidad. Madrid: Editorial Losada.

Bourdieu, P. (1997) [1994]. Raisons Pratiques. Francia: Seuil.

Caggiano, S. (2005): Lo que no entra en el crisol. Inmigración boliviana, comunicación intercultural y procesos identitarios. Buenos Aires : Prometeo. 
Castells, M. (1997) La era de la información: economía, sociedad, cultura. El poder de la identidad. Buenos Aires: Alianza.

CELADE: 1979, "El problema del éxodo del personal calificado en la América Latina, diagnóstico y políticas", en Cuadernos del CELADE, $\mathrm{N}^{\circ}$ 2, Santiago de Chile.

Cerrutti, M. y Maguid, A. (2007): "Inserción laboral e ingresos de migrantes limitrofes y del Perú en el Área Metropolitana de Buenos Aires, 2005", (en línea) http://www.eclac.org/celade/noticias/paginas/5/27255/ Cerrutti_Maguid.pdf, acceso el 17 de octubre de 2011.

Chacon Avila, L. "Reflexiones sobre la migración de recursos humanos calificados: ", Amérique Latine Histoire et Mémoire. Les Cahiers ALHIM, 5 | 2002, (en línea). http://alhim.revues.org/index700.html, acceso el 19 de noviembre de 2008.

Domenech, E. (2005). Migraciones contemporáneas y diversidad cultural en la Argentina. Córdoba: Centro de Estudios Avanzados de la Universidad Nacional de Córdoba.

Halpern, G. (2009): Etnicidad, inmigración y politica. Representaciones y cultura politica de exiliados paraguayos en Argentina. Buenos Aires: Prometeo.

Levitt, P. y Glick Schiller, N. (2004): "Perspectivas internacionales sobre migración: Conceptualizar la simultaneidad" en Migración y Desarrollo, $\mathrm{N}^{\circ} 3$, segundo semestre, 60-91.

Maguid, A. y Bruno, S. (2010): "Migración, mercado de trabajo y movilidad ocupacional: el caso de los bolivianos y paraguayos en el Área Metropolitana de Buenos Aires", en Población de Buenos Aires. Revista semestral de datos y estudios sociodemográficos urbanos, Buenos Aires: Dirección General de Estadística y Censos (DGEyC) del Gobierno de la Ciudad de Buenos Aires. 7-28. 
Mármora, L. (2002). Las politicas de Migraciones Internacionales OIM. Buenos Aires: Paidós.

Martiarena, M.L. (1998): "Sistemas Regionales de Ciencia, Técnica e Innovación", Panel sobre Políticas Regionales del Seminario Nacional de debate del Proyecto Plurianual de Ciencia y Tecnología 1999 - 2001, Buenos Aires.

Mato, D. (2007): "Cultura, comunicación y transformaciones sociales en tiempos de globalización", en Cultura y transformaciones sociales en tiempos de globalización, CLACSO.

Massey, D. (2005): "La filosofia y la política de la especialidad: algunas consideraciones". En L. Arfuch (comp.), Pensar este tiempo. Espacios afectos, pertenencias. Buenos Aires: Paidós. Pp. 101-127.

Mera C. (1998): La inmigración coreana en Buenos Aires. Multiculturalismo en el espacio urbano. EUDEBA, Buenos Aires.

Mera, C. (2005): "Migración coreana: identidades entre desplazamientos y anclajes", en Cohen, N. y Mera, C. (comp.) Relaciones Interculturales: experiencias $y$ representación social de los migrantes. Buenos Aires: Antropofagia, pp. 9-24.

Mera, C. (2007): Globalización e identidades migrantes. Corea y su diáspora en la Argentina, tesis de doctorado de UBA (en prensa)

Miurbal (2007) Boletín del Observatorio, N2 Diciembre 2007, http://www.miurbal.net

Ortiz, R. (2003): Lo próximo y lo extraño. Japón y la modernidad-mundo, Interzona, Buenos Aires.

Oteiza, E. (1976): "El drenaje de Cerebros", en Términos Latinoamericanos para el Diccionario de Ciencias Sociales, CLACSO - ILDIS, Buenos Aires. 
Pacecca, M. I. (2000): "Legislación, migración limítrofe y vulnerabilidad social", en Realidad Económica, núm. 171, Buenos Aires, abril-mayo, pp 111-134.

Sassen, S. (1999): La ciudad global, Nueva York, Londres, Tokio, EUDEBA.

Sassen, S. (2003): Los espectros de la globalización, FCE, Buenos Aires

Sassone, S. M. y Mera, C. (2007): "Barrios de migrantes en Buenos Aires: Identidad, cultura y cohesión socioterritorial". En Las relaciones triangulares entre Europa y las Américas en el siglo XXI: expectativas y desafios. Bruselas: Ponencia presentada en el V Congreso Europeo CEISAL de latinoamericanistas, 11 al 14 de abril.

Wallerstein, I. (2001). Conocer el mundo. Saber el mundo. El fin de lo aprendido. Una ciencia social para el siglo XXI. México D. F: Siglo XXI Editores.

\section{FORMA DE CITAR ESTE TRABAJO EN BIBLIOGRAFÍAS HOW TO CITE THIS ARTICLE IN BIBLIOGRAPHIES}

Mera, C. y Halpern, G. (2011): "Migraciones internacionales: repensando las ciudades y sus politicas", Revista Latina de Sociología, n ${ }^{\circ}$ 1: 98-125, http://revistalatinadesociologia.com, ISSN 2253-6469 